\title{
Influence of Modifiers on Supercritical Fluid Chromatography (SFC) and Supercritical Fluid Extraction (SFE), Part I
}

\author{
Ilia Brondz ${ }^{*}$, Boris Sedunov ${ }^{2}$, Nagarajan Sivaraman ${ }^{3}$ \\ ${ }^{1}$ Norwegian Drug Control and Drug Discovery Institute (NDCDDI) AS, Ski, Norway \\ ${ }^{2}$ The Computer and Information Systems Department of the Russian New University, Moscow, Russia \\ ${ }^{3}$ Fuel Chemistry Division, Indira Gandhi Centre for Atomic Research (IGCAR), Kalpakkam, India \\ Email: ^ilia.brondz@gmail.com, sedunov.b@gmail.com, sivaram@igcar.gov.in
}

How to cite this paper: Brondz, I., Sedunov, B. and Sivaraman, N. (2017) Influence of Modifiers on Supercritical Fluid Chromatography (SFC) and Supercritical Fluid Extraction (SFE), Part I. International Journal of Analytical Mass Spectrometry and Chromatography, 5, 17-39.

https://doi.org/10.4236/ijamsc.2017.52002

Received: April 5, 2017

Accepted: June 27, 2017

Published: June 30, 2017

Copyright $\odot 2017$ by authors and Scientific Research Publishing Inc. This work is licensed under the Creative Commons Attribution International License (CC BY 4.0).

http://creativecommons.org/licenses/by/4.0/

\begin{abstract}
It is important to understand the mechanism and implications of different modifiers on analytical and preparative processes under chromatography with supercritical fluids (SFs) and under extraction with SFs. Supercritical fluid chromatography (SFC) and supercritical fluid extraction are generally carried out with neat supercritical carbon dioxide $\left(\mathrm{SCCO}_{2}\right)$ or with $\mathrm{SCCO}_{2}$ containing modifiers (or cosolvents), especially for strongly polar compounds. For example, methanol is added as a cosolvent/modifier to $\mathrm{SCCO}_{2}$ for the extraction/separation of polar compounds. This paper discusses the influence of the modifier on the colligative properties of the principal mobile phase, which may define the situation in the total mobile phase in a chromatography column or in parts of a column under SFC. No colligative behavior of solutions reflects individual properties of the solutes. Their cross-interactions with solvents are discussed.
\end{abstract}

\section{Keywords}

Supercritical Fluid (SF), Supercritical Fluid Chromatography (SFC), Supercritical Fluid Extraction (SFE), Modifiers, Influence of Modifiers, Colligative Properties, Thermodynamics-Based Evaluation of Binary Mixtures, Phase Equilibria

\section{Introduction}

There are two main components of supercritical fluid chromatography (SFC) instrumentation: a capillary column supercritical fluid chromatograph, derived from a gas chromatograph instrument, and a packed column supercritical fluid 
chromatograph, derived from a high-performance liquid chromatograph instrument [1] [2] [3]. The mobile phase is delivered to the instrumentation used for capillary column supercritical fluid chromatography (CCSFC) as an unmodified or a modified supercritical fluid (SF) directly from a storage cylinder. CCSFC is, however, very seldom used. The focus of this paper is on packed column supercritical fluid chromatography (PCSFC), and we discuss its theoretical background.

The literature describes the reasons for failures in the construction of capillary column supercritical fluid chromatographs and CCSFC procedures, which make use of delivery from a single cylinder with premixed supercritical carbon dioxide $\left(\mathrm{SCCO}_{2}\right)$ and a modifier (methanol or formic acid) [4]. Several PCSFC concepts and procedures in which the modifier is mixed with $\mathrm{SCCO}_{2}$ by a server pump in the mixer chamber are also described. Comprehensive reviews of the development and use of SFC techniques, methods, and instrumentation are presented [1]-[10].

SFC is a very promising analytical tool for demanding separations of complex mixtures and isomers [1] [3] [5]-[10]. However, both the hardware, such as instrumentation and columns, and the theoretical basis are underdeveloped, in comparison with high-performance liquid chromatography (HPLC) and gas chromatography. A somewhat better situation exists for supercritical fluid extraction (SFE) [11]. The most attractive technique is PCSFC, largely due to the several advantages it offers: a wide range of detectors may be used [5] [10], it is possible to use pressure programming and temperature programming, and there is a wide choice of modifiers available, in isocratic and programmed modes, for altering the composition of the mobile phase, pressure, and temperature. Using PCSFC, it is possible to perform semipreparative and preparative fractionation; this possibility is very limited, or impossible, with CCSFC.

The most attractive and frequently employed substance for SFE, as well as for SFC, is $\mathrm{CO}_{2}$ in its supercritical state. $\mathrm{SCCO}_{2}$ possesses favorable properties: it is noncorrosive, nonflammable, nontoxic, and environmentally friendly.

Some other inorganic candidates that may be employed, but are seldom used as principal mobile phases in a supercritical state, are xenon (Xe), nitrous oxide $\left(\mathrm{N}_{2} \mathrm{O}\right)$, sulfur hexafluoride $\left(\mathrm{SF}_{6}\right)$, ammonia $\left(\mathrm{NH}_{3}\right)$, water $\left(\mathrm{H}_{2} \mathrm{O}\right)$, and freons. Alkanes, such as ethane $\left(\mathrm{C}_{2} \mathrm{H}_{6}\right)$, propane $\left(\mathrm{C}_{3} \mathrm{H}_{8}\right)$, and $n$-butane $\left(\mathrm{C}_{4} \mathrm{H}_{10}\right)$ have also been used, but they are highly flammable, making them the least preferred candidates for mobile phases.

Physicochemical properties and characteristics of polar and nonpolar SFs that may be used in SFE and SFC technologies are presented in the Table 1 (reproduced from [12]).

\subsection{Carbon Dioxide as the Principal Mobile Phase for SF Technologies}

Figure 1 presents the phase diagram for pressure-temperature relations (bar/ Kelvin, $\mathrm{K}$ ) for neat $\mathrm{CO}_{2}$, with its triple and critical points. Built on thermophysical data from the NIST databases [13] [14], Figure 1 develops the idea of ex- 
Table 1. Characteristics of selected supercritical fluids.

\begin{tabular}{cccc}
\hline Fluid & $T_{c}\left({ }^{\circ} \mathrm{C}\right)$ & $P_{c}(\mathrm{~atm})$ & Dipole moment (Debye) \\
\hline $\mathrm{CO}_{2}$ & 31.3 & 72.9 & 0.00 \\
$\mathrm{~N}_{2} \mathrm{O}$ & 36.5 & 72.5 & 0.17 \\
$\mathrm{NH}_{3}$ & 132.5 & 112.5 & 1.47 \\
$\mathrm{Methanol}$ & 240.0 & 78.5 & 1.70 \\
$\mathrm{Xe}$ & 16.6 & 58.4 & 0.00 \\
$\mathrm{CCI}_{2} \mathrm{~F}_{2}$ & 111.8 & 40.7 & 0.17 \\
$\mathrm{CCIF}_{3}$ & 28.8 & 38.2 & 0.50 \\
Ethane & 32.2 & 48.2 & 0.00 \\
\hline
\end{tabular}

Many other substances also can be used http://www.supercriticalfluid.org/Supercritical-fluids.146.0.html.

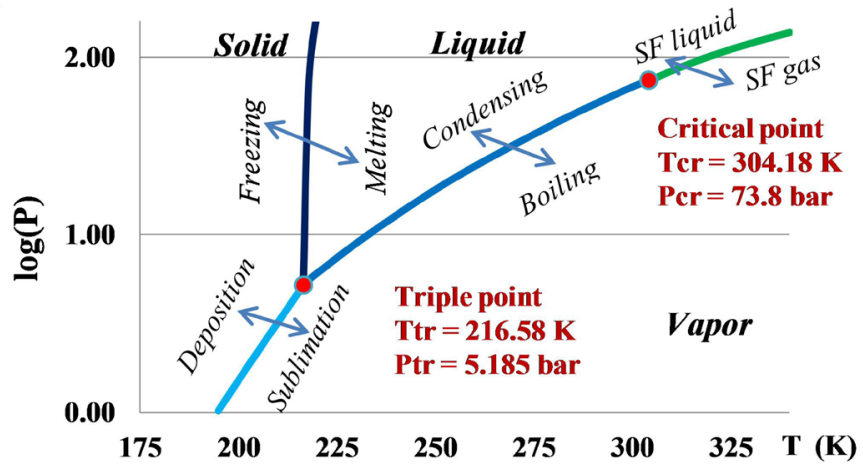

Figure 1. Pressure-temperature relations (bar/Kelvin, $\mathrm{K}$ ) in pure $\mathrm{CO}_{2}$, built on data from the NIST databases [13] and [14].

tending the saturation line to the SF zone, where a soft structural transition from the gas-like SF to the liquid-like SF takes place at a fluid density close to the critical density $\mathrm{D}_{\mathrm{c}}$ [15] [16]. This extension is known as the Widom line. The equation for sublimation vapor pressure is taken from the NIST database [14].

The phase diagrams indicate the conditions for triple and critical points; the regions for the existence of vapor, liquid, condensing, melting, freezing, existence of solid, deposit, and sublimation; and the lines of sublimation, melting, and saturation. The important conditions for the critical point and the extension of the saturation line to the SF zone are also evident.

The influence of modifiers on phase diagrams for pressure-temperature relations and the triple and boiling points, and lines of solid, liquid, and vapor separation will be demonstrated later.

\subsection{The Role of Modifiers to $\mathrm{CO}_{2}$ in SF Technologies}

Neat $\mathrm{CO}_{2}$ in a supercritical state is primarily employed for the extraction of hydrophobic molecules and, at best, it can dissolve some moderately polar compounds. $\mathrm{CO}_{2}$ alone is very seldom used. This is because neat $\mathrm{CO}_{2}$ is rather lipophilic and polar/strong polar substances do not dissolve well in it. This issue can be resolved to some extent by elevation of pressure or modification of temperature, or both [3]. In addition to altering the pressure and temperature of neat 
$\mathrm{CO}_{2}$, modifiers or cosolvents-which are polar in nature, such as water, methanol, ethanol, diethylamine, formic acid, acetic acid, and many other polar and even nonpolar solvents, and complexation agents-are added to $\mathrm{SCCO}_{2}$ to enhance bond separation and the extraction of strongly polar compounds and ions. By using one component as modifier, or a mixture of different substances as modifiers, it is possible to achieve a wide range of analyte solubilities in the mobile phase of modified $\mathrm{SCCO}_{2}$. Methanol and ethanol are often used as modifiers in SFC to enhance mobile phase polarity. Small $\mathrm{m} / z$ ions from these modifiers often do not disturb the fragmentation picture of large molecules under SFCmass spectrometry (MS).

Methanol is an important modifier for $\mathrm{SCCO}_{2}$ to achieve some polarity in this mobile phase [17]. However, ethanol is less toxic for the operator and the environment; it has been used in up to $30 \%$ molar volume in isocratic mode [18]. The practical experiments and data described in [19] are considered very important because of the large volumes of ethanol used as modifier. Multicomponent mixtures of $\mathrm{CO}_{2}$, ethanol, and diethylamine, described in [18], are not unique. Many complicated multicomponent mixtures of $\mathrm{CO}_{2}$ with modifiers [17], cosolvents, and even complexation agents are in use [11].

Calculation of phase equilibria for multicomponent mixtures as described by Gernert et al. [20] can be very helpful for understanding the processes in SFC columns during bond separation in complex mixtures or the resolution of isomers. In section of columns under separation together with complex mobile phase also present analytes of different nature (which are present in initial analyzing mixture). Chueh and Prausnitz [21] [22] have modeled $\mathrm{CO}_{2}$ with mixtures of some of these compounds (including methanol and ethanol) for the estimation of the critical parameters $T_{c}$ and $P_{c}$. The most complete collection of $\mathrm{SCCO}_{2}$ solubility data with multiple solutes is presented in "Solubility in Supercritical Carbon Dioxide" by Ram B. Gupta and Jae-Jin Shim [23].

In some cases, polar supercritical phases have been used for chromatography and extraction and have advantages over $\mathrm{CO}_{2}$ in the supercritical state [24]. This provides quantitative recovery and the possibility to overcome the matrix-analyte interaction, which sometimes may be more important than a high solubility of the analyte in the solvent [24]. Polar supercritical principal phases, such as ammonia, can be used for the extraction of metal ions or polar substances from matrices by SFE or SFC [25]. Many salts and substances are readily soluble in ammonia in the supercritical state and this principal phase can also be modified to lend the principal mobile phase some lipophilic characteristics [25]. The pressure-temperature diagram for ammonia is presented in Figure 2, built on thermophysical data from the NIST databases [13] and [14].

In several cases, ammonia in the supercritical state has advantages because it is polar, in contrast to $\mathrm{CO}_{2}$ containing polar modifiers for the extraction of strongly polar compounds. This is because the addition of polar modifiers to $\mathrm{CO}_{2}$ results in alteration of the critical temperature and critical pressure. Hence, the integrity of the supercritical phase should be carefully preserved by choosing 


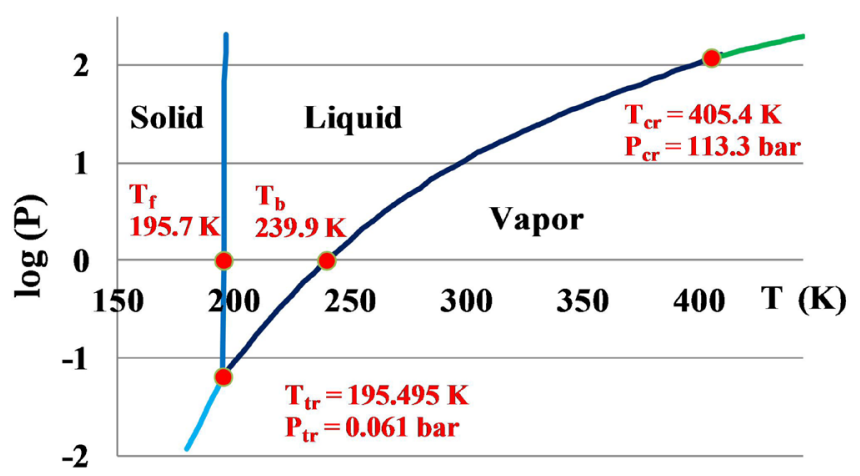

Figure 2. The pressure-temperature diagram for ammonia, built on data from the NIST databases [13] and [14]. Round red markers show freezing and boiling points at $P=1 \mathrm{bar}$, critical and triple points. The green line to the right of the critical point demonstrates the Widom line extension of the saturation line to the supercritical fluid zone.

appropriate experimental pressures and temperatures. However, ammonia is extremely corrosive and toxic, which makes it the least preferred mobile phase.

\subsection{Phase Transformations in SFC Columns}

The mobile phase in a chromatography column under SFC is not homogeneous, even when using neat $\mathrm{CO}_{2}$, because of a pressure gradient fall from the head of the column to the bottom (outlet). However, modern SFC instrumentation is equipped with a backpressure regulator (BPR) that can automatically hold a constant pressure at the outlet of the column in programmed mode, and hence define the pressure at the head of the column. The main purpose of a BPR is to prevent the conversion of $\mathrm{SCCO}_{2}$ to $\mathrm{CO}_{2}$ gas at the outlet of the column as well as to prevent freezing at the outlet as a result of this, i.e., the analyte is kept in the mobile phase in the supercritical state until it arrives in the detector.

In real situations, the following are often present in a column: in the head section of the column, the $\mathrm{CO}_{2}$ mobile phase in a liquid state (depending on conditions); in the middle of the column, a section of $\mathrm{SCCO}_{2}$; while at the bottom of the column, there is the section where $\mathrm{CO}_{2}$ often exists as condensed gas under a high pressure (as a fluid under subcritical conditions). Of course, the operator can achieve better conditions by using an isocratic mode of operation with only two different phases: liquid in the head section of the column and SF in the main part of the column. However, this is not achievable with a programmed mode of operation of conditions and parameters, such as programmed pressure, programmed temperature, and programmed concentration of modifier in the mobile phase, simultaneously.

\subsection{Use of Cosolvents/Modifiers in SFE}

The solvating power of a SF at a given temperature depends on its density. The solubility of a solute in $\mathrm{SCCO}_{2}$ depends on the intermolecular interactions between solvent and solute. The solvating power of $\mathrm{SCCO}_{2}$ can be defined in terms 
of the solubility parameter, $\delta$. It is defined as the square root of the cohesive energy density and is used extensively in interpreting the thermodynamic properties of a solution. It is given as follows:

$$
\begin{gathered}
\delta=C^{1 / 2}, \\
C=(\Delta H-R T) / V .
\end{gathered}
$$

where $\Delta H$ is the heat of vaporization, $V$ is the molar volume of the liquid, and $C$ is the cohesive energy density.

For a SF, the heat of vaporization is zero at the critical temperature; therefore, the above equation leads to a negative value of $C$. The equation was later modified and Giddings proposed an equation based on studies in liquid chromatography using the van der Waals equation of state. The $\delta$ for a SFC is given as follows:

$$
\delta=1.25 P_{C}^{1 / 2} \frac{\rho_{r}}{\rho_{r, L I Q}}
$$

where $\rho_{r}$ is the reduced density of the substance in supercritical state and $\rho_{r, l i q}$ is the reduced density of the liquid at its normal boiling point.

Thus, the solubility parameter for a given substance can be obtained if the fluid density and critical pressure are known, and it is unique for a given substance. When the solubility parameters of both the solute and solvent are similar, appreciable solubility of the solute will generally be observed in the given solvent. The solubility parameters of some SFs are given.

$\mathrm{CO}_{2}$ in supercritical state is a nonpolar medium and it finds wide application in the extraction of organic compounds, such as natural products, which are generally hydrophobic in nature. $\mathrm{SCCO}_{2}$ with the addition of modifiers/cosolvents, such as methanol, is used for extraction of polar compounds. SFE of metal ions can be performed by adding suitable chelating agents to the $\mathrm{SCCO}_{2}$ medium.

Modifiers or cosolvents are the substances that are added to SFs to alter solvent properties, i.e., to increase the polarity, aromaticity, chirality and the ability to complex various metal ions. For example, methanol or ethanol is added to $\mathrm{CO}_{2}$ to increase polarity; toluene to impart aromaticity and neutral extractant; tri- $n$-butyl phosphate, dialkylalkyl phosphonates, dibutylbutyl phosphonate, and

Table 2. Solubility parameters of fluids at supercritical state.

\begin{tabular}{ccc}
\hline Compound & $\begin{array}{c}\text { Solubility parameter } \boldsymbol{\delta} \\
\left(\mathrm{cal} / \mathrm{cm}^{3}\right)^{1 / 2}\end{array}$ & $\begin{array}{c}\text { Solubility parameter } \boldsymbol{\delta} \\
\left(\mathrm{MPa}^{1 / 2}\right)\end{array}$ \\
\hline Ammonia $\left(\mathrm{NH}_{3}\right)$ & 13.2 & 27.2 \\
Carbon dioxide $\left(\mathrm{CO}_{2}\right)$ & 10.7 & 22 \\
Nitrous oxide $\left(\mathrm{N}_{2} \mathrm{O}\right)$ & 10.6 & 21.8 \\
Xenon $(\mathrm{Xe})$ & 9.5 & 19.5 \\
Propane $\left(\mathrm{C}_{3} \mathrm{H}_{8}\right)$ & 8.1 & 16.7 \\
$n$-Hexane $\left(\mathrm{C}_{6} \mathrm{H}_{14}\right)$ & 7.0 & 14.4 \\
\hline
\end{tabular}


diamylamyl phosphonate for solvation of metal complexes [11]. Addition of polar cosolvents may be effective in solubilizing the polar solute molecules. The modified $\mathrm{CO}_{2}$ solvent may also absorb on the active sites of the matrix and prevent the readsorption of the analyte, and thereby its transfer to the supercritical phase. When modifiers are added to SFs, it is essential to evaluate the phase equilibria of the mixture and chemical interactions between them. Issues relating to estimation of the critical parameters for the mixtures should be carefully evaluated. At the critical point, the composition and density of the two phases become identical, and only one SF phase exists. For a given mixture at a given temperature, there will be a critical pressure and critical composition value.

Knowledge about the modifier-fluid phase diagram is essential to ensure that the solvent is in a single phase. For binary mixtures with a low interaction between components, the critical constants are approximately the arithmetic means of the $T_{C}$ and $P_{C}$.

$$
\begin{aligned}
& T_{C}=x_{A} T_{A}+x_{B} T_{B} \\
& P_{C}=x_{A} P_{A}+x_{B} P_{B}
\end{aligned}
$$

Here $x_{A}$ and $x_{B}$ are the molar shares of components $A$ and $B$ in the mixture. $T_{A}$ and $T_{B}$ are the critical temperatures and $P_{A}$ and $P_{B}$ are the critical pressures of components $A$ and $B$, respectively.

In some mixtures, the cosolvent can interact with $\mathrm{CO}_{2}$ and the resultant $T_{C}$ and $P_{C}$ may differ from the values based on a linear combination of the components $T_{C}$ and $P_{C}$. Brunner et al. [26] estimated the critical parameters for the methanol- $\mathrm{CO}_{2}$ system. $\mathrm{CO}_{2}$ containing methanol can be taken to critical point only above a certain temperature, depending upon the composition of methanol in the $\mathrm{CO}_{2}$ phase. For the methanol- $\mathrm{CO}_{2}$ system at the $\mathrm{CO}_{2}$ molar share equal to 0.84 , the critical temperature is $50^{\circ} \mathrm{C}$ and the critical pressure is 95.5 bar. However, linear estimation for the critical temperature of the above mixture is around $64^{\circ} \mathrm{C}$ and linear estimation for the critical pressure is around 75 bar.

Because direct extraction of ionic species is inefficient in a neat $\mathrm{SCCO}_{2}$ medium, chelating agents are generally added to a $\mathrm{SCCO}_{2}$ medium to form neutral complexes. The latter are quite soluble in supercritical medium. A variety of chelating agents such as organophosphorous compounds, dithiocarbamates, and macrocyclic ligands have been used for the extraction of metal ions such as lanthanides, actinides, and transition metal ions [27]-[34]. Tributylphosphine (TBP) and trioctylphosphine oxide (TOPO) are some of the solvating extractants used in the SFE of actinides. Crown ethers are used for selective extraction of alkali/alkaline earth metal ions. The solubility of metal complexes in SFC medium is an important criterion for designing an efficient metal extraction process. In general, many chelating agents show relatively high solubility in $\mathrm{SCCO}_{2}$. The $\beta$-diketones and fluorinated diketones have been used in $\mathrm{SCCO}_{2}$ medium for the SFE of lanthanides and actinides. The extraction efficiency depends on the solubility of ligands as well as that of metal complexes in the supercritical medium.

In some cases, $\mathrm{SCCO}_{2}$ will be delivered to an extraction vessel containing the ligand. The $\mathrm{SCCO}_{2}$ stream containing the ligand is subsequently passed through 
another vessel containing the analyte (metal ion of interest). On some occasions, ligand is dissolved in methanol medium and mixed into $\mathrm{SCCO}_{2}$ and the modified stream will be employed for metal ion extraction.

\section{Colligative Properties of $\mathrm{CO}_{2}$ with Modifiers}

Colligative properties, such as vapor pressure depression, boiling point elevation, melting/freezing point depression, and osmotic pressure have played a decisive role in analytical chemistry prior to the discovery of chromatography and MS for identification of purity as well as for the determination of the molecular weight of substances. Use of the melting/freezing point depression of camphor or benzene was nearly a standard test for determining the molecular weight of an unknown substance.

Colligative properties depend only on the molar share $y$ of the solute in solution, not on the nature of the solute. The molecular weight of the unknown nonvolatile substance is calculated from melting/freezing point depression $\Delta T_{f}$ using the following formula:

$$
\Delta T_{f}=i K_{f} m
$$

where $K_{f}$ is the cryoscopic constant, $\Delta T_{f}$ is the melting temperature depression, and $m$ is the molality.

Van't Hoff factor $i$ is the number of particles that may arise from one solute molecule at its possible dissociation. In a nonpolar liquid or $\mathrm{SCCO}_{2}$, there are no conditions for dissociation of solute molecules, and the van't Hoff factor $i=1$. The molality $m$ is the number of moles of solute in $1 \mathrm{~kg}$ of a solvent [35]:

$$
m=1000 y /(M(1-y)) \text {. }
$$

where $M$ is the gram molecular weight of the solvent and $y$ is the molar share of solute. From Equations (1) and (2), we can determine the cryoscopic constant $K_{f}$.

$$
K_{f}=\Delta T_{f} / m=\Delta T_{f}(M(1-y)) /(1000 y) .
$$

At low values of $y$, the Equation (3) takes a more simple form: $K_{f}=D T_{f} M /(1000 y)$.

For a solution of a nonvolatile solute with molar share y, Raoult's law [35] gives us the vapor pressure of solvent $P_{\text {sol }}=P_{\text {sat }}(1-y)$, where $P_{\text {sat }}$ is the vapor pressure of pure solvent. The triple point temperature depression results from derivations of $P_{\text {sub }}(T)$ and $P_{\text {sat }}(T)$ :

$$
\Delta T_{t r}=P_{t r} y /\left(\mathrm{d} P_{\text {subl }} / \mathrm{d} T-(1-y) \mathrm{d} P_{\text {sat }} / \mathrm{d} T\right) .
$$

where $P_{t r}$ is the triple point vapor pressure, and $P_{s u b l}(T)$ and $P_{s a t}(T)$ are vapor pressures along the sublimation and saturation lines, respectively. Equation (4) may be rewritten as:

$$
\Delta T_{t r}=R T^{2} y /\left(H_{\text {subl }}-(1-y) H_{\text {sat }}\right) .
$$

where $H_{\text {subl }}$ and $H_{\text {sat }}$ are sublimation and saturation enthalpies for solid and liq- 
uid solvent.

Upon adding a substance (of another nature) to a pure solvent, we will achieve new properties, such as the melting, boiling, vaporization, condensing, freezing, triple, and critical points for this mixture, in comparison with the properties of the initial pure solvent. In the case of $\mathrm{CO}_{2}$ in the supercritical state, upon adding the analyte as modifier we obtain a new pressure-temperature diagram (bar/ Kelvin, $\mathrm{K}$ ) in modified $\mathrm{CO}_{2}$. However, $\mathrm{CO}_{2}$ differs from other solvents due to the high value of its triple point pressure, precluding the standard determination of melting and boiling points.

Despite an extensive search of the literature for the crucial parameters for the calculation of a new diagram of pressure-temperature relations for modified $\mathrm{CO}_{2}$, we have not yet found the cryoscopic constant $K_{f}$ for $\mathrm{CO}_{2}$, because the triple point pressure in $\mathrm{CO}_{2}$ is much above 1 bar. For this reason, we decided to use qualitative changes only as a model for understanding changes. When a nonvolatile solute is added to a solvent, the resulting solution has a vapor pressure $P_{s o l}(T)$ that is lower than the pure solvent saturation pressure $P_{\text {sat }}(T)$, in accordance with the laws of Raoult and Dalton [35].

The diagram for qualitative changes in pressure-temperature relations in $\mathrm{CO}_{2}$ with a nonvolatile and nondissociating modifier at molar share $y=0.1$ is presented in Figure 3, built on experimental data from the NIST databases [13] and [14].

Bearing in mind that in pure $\mathrm{CO}_{2}$ the triple point pressure $P_{t r}=5.185 \mathrm{bar}$, we had to take (in this unusual case) the value of pressure $P_{b}$ for the estimation of melting and boiling points 10 times higher than the normal pressure. Hence, we investigated the $\mathrm{CO}_{2}$ boiling point at an elevated pressure $P_{b}=10$ bar. Figure 3 shows that in this case the depression of the melting point $\Delta T_{f}$ is almost the same as the $\Delta T_{t r}$ at $3.1 \mathrm{~K}$. Estimation of the $\Delta T_{t r}$ based on Equation (4), almost coincides with this value.

Equation (3) gives us an estimation for the cryoscopic constant in $\mathrm{CO}_{2}, K_{f}=$

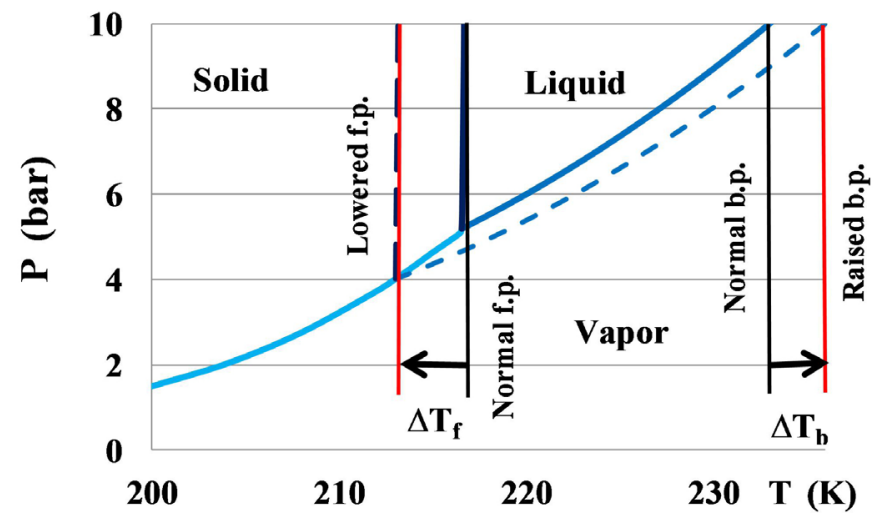

Figure 3. Theoretical diagram for changes (dashed lines) in pressure-temperature relations in $\mathrm{CO}_{2}$ with a nonvolatile and nondissociating modifier at molar share $y=0.1$ in solution. The freezing point (f.p.) and boiling point (b.p.) are estimated for elevated pressure $P_{b}=$ 10 bar. The data for pure $\mathrm{CO}_{2}$ are based on [13] and [14]. 
$1.23 \mathrm{~K} \mathrm{~kg} / \mathrm{mol}$. This value is low, compared with other known solvents, and is much smaller than the $K_{f}$ for water, which is $1.853 \mathrm{k} \cdot \mathrm{kg} / \mathrm{mol}$. This may be explained by a large difference between $H_{\text {subl }}$ and $H_{\text {sat }}$ in $\mathrm{CO}_{2}$ near the triple point. Elevated bond energy between $\mathrm{CO}_{2}$ molecules in the solid phase prevents from melting up to high enough $T_{t r}$ temperature. For this reason, the ratio of triple point temperature to critical temperature $T_{t r} / T_{c r}=0.712$ is uniquely large for $\mathrm{CO}_{2}$ compared with other substances. This large $T_{t r} / T_{c r}$ ratio also explains the uniquely large $P_{t r}$ value for $\mathrm{CO}_{2}$.

To estimate the increase in boiling temperature in a $0.1 \mathrm{M}$ solution, we built the saturated vapor pressure $P_{\text {sat }}(T)$ dependence for pure $\mathrm{CO}_{2}$ (solid blue line in Figure 3, based on data from [13]), and the partial $\mathrm{CO}_{2}$ vapor pressure $P_{y}(T)$ over a $0.1 \mathrm{M}$ solution (dashed blue line), which according to Raoult's law is equal to $P_{y}(T)=P_{\text {sat }}(T)(1-y)$. We found temperature $T_{\text {sat, }}$ corresponding to pure $\mathrm{CO}_{2}$ at pressure $10 \mathrm{bar}$, and $T_{y}$, corresponding to $0.1 \mathrm{M}$ solution at the same pressure: $T_{\text {sat }}=233.03 \mathrm{~K}$ and $T_{y}=235.96 \mathrm{~K}$. The difference is the boiling temperature elevation $\Delta T_{b}=2.93 \mathrm{~K}$.

The ebullioscopic constant may be calculated from the boiling temperature elevation $\Delta T_{b}$ using a formula, similar to Equation (3):

$K_{b}=\Delta T_{b} / m=\Delta T_{b}((1-y) M) /(1000 y)$. Figure 3 and $P_{\text {sat }}(T)$ dependence give an estimation for the boiling temperature $\Delta T_{b}$ elevation in $\mathrm{CO}_{2}$ for $0.1 \mathrm{M}$ solution at boiling pressure $P_{b}=10 \mathrm{bar}, \Delta T_{b}=2.93 \mathrm{~K}$. This results in $K_{b}=1.16$ $\mathrm{kg} / \mathrm{mol}$. This value is close to $K_{b}=1.19 \mathrm{~K} \mathrm{~kg} / \mathrm{mol}$ for ethanol, but was found at 10 times higher pressure.

\section{Solubility of Polar Modifiers in Dense Gaseous Solvents}

In studying polar modifiers, such as water, methanol, or ethanol, added to solvents, as used in SF technologies, we start from dense gaseous solutions. The dimension of solubility is $\mathrm{mol} / \mathrm{mol}$ : the number of solute moles per one mole of the gas phase. The solubility mechanism in the range of pressure $P$ under one-half of the solvent's critical pressure $P_{c}$ may be easily understood because, at these pressures, the structure of a mixture is very simple: the gaseous mixture of solver and solute fractions with the dimers' fraction, resulting from the crossinteraction between solver and solute molecules.

At $P<P_{d} / 2$ the dominating mechanism of solution is filling of the solution molar volume $V=R T / P$ by solute molecules. We refer to this type of solubility as solubility in a free space, created by the solvent molecules, for distribution of the solute molecules with their saturation vapor density.

Experimental data of Coan and King [36] and Wiebe [37] for equilibrium solubility of water in gaseous $\mathrm{CO}_{2}, \mathrm{~N}_{2} \mathrm{O}$, and $\mathrm{C}_{2} \mathrm{H}_{6}$ demonstrate that the solubility at pressures lower than one-half of the solvent's critical pressure decreases with pressure, then passes through a minimum, and at supercritical pressures increases with pressure (see Figure 4). This paradoxical behavior of solubility has attracted the attention of researchers.

At a fixed subcritical temperature, the minimum water solubility in $\mathrm{CO}_{2}$ is at a 


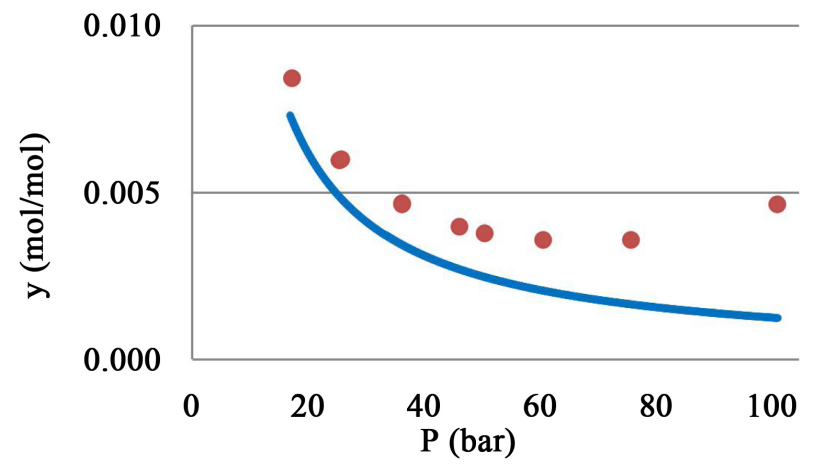

Figure 4. Water solubility $y$ in gaseous $\mathrm{CO}_{2}$ according to experimental data from [36] (markers) and theoretical free space solubility $y_{f s}$ (solid blue line) at supercritical temperature $50^{\circ} \mathrm{C}$.

pressure value slightly less than the solvent's saturation pressure $P_{\text {sat }}(T)$. At supercritical temperatures, it tends toward the gas-to-liquid transition line [15], known as the Widom line. This behavior of solubility in subcritical and supercritical $\mathrm{CO}_{2}$ is known for many other gaseous solute-solvent systems [23].

Coan and King have explained the solubility pressure dependence in these systems by the liquid phase fugacity dependence on pressure and by high crossinteraction virial coefficients for the molecular interaction of water with gaseous solvents [36]. A higher than the dispersion forces, the cross-interaction of water with $\mathrm{CO}_{2}$ or $\mathrm{N}_{2} \mathrm{O}$ solvents they explain by quadrupole moments [38] of $\mathrm{CO}_{2}$ and $\mathrm{N}_{2} \mathrm{O}$ molecules. The symmetry of ethane molecules forbids not only the dipole moment but also the quadrupole moment. But it does not mean that the molecular interaction of water with ethane has a pure dispersion mechanism. The charged $\mathrm{C}$ and $\mathrm{H}$ atoms in an ethane molecule, discussed in [39], create the multipole moment [38], which can explain the enforced cross-interaction of ethane molecules with molecules of polar solutes.

We suggest a more obvious scheme for the water solution in these solvents, one that presents three main types of the solute-solvent interaction, dominating or essential in different solvent pressure ranges, as demonstrated in Figure 5 for the $\mathrm{H}_{2} \mathrm{O}-\mathrm{CO}_{2}$ system.

\subsection{Water Solubility in Gaseous $\mathrm{CO}_{2}$ at Pressure below $\boldsymbol{P}_{c} / 2$}

At a solution pressure $P$ lower than $P_{d} / 2$, the following dominates: distribution of solute molecules in a free space of a gaseous solution $V_{f_{5}}(T, P)$ approximately equal to the solvent's molar volume $V_{s}(T, P)$. The larger the $V_{s}(T, P)$, the more moles of solute vapor may be distributed in this volume. The resulting contribution of this dominating mechanism in the solubility coefficient $y_{f s}$ (Figure 6) is equal to:

$$
y_{f s}=V_{f s}(T, P) D_{\text {wsat }}(T) .
$$

where $D_{\text {wsat }}(T)$ is the saturated water vapor molar density at temperature $T$. It can be taken from the NIST database [13].

The solvent's molar volume $V_{s}(T, P)$ increases at low pressures as $V_{s}(T, P)=$ 


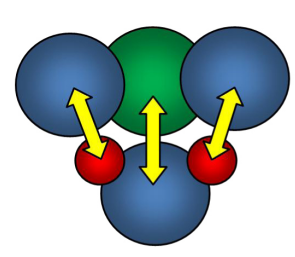

Solute - Solvent Molecular Interaction

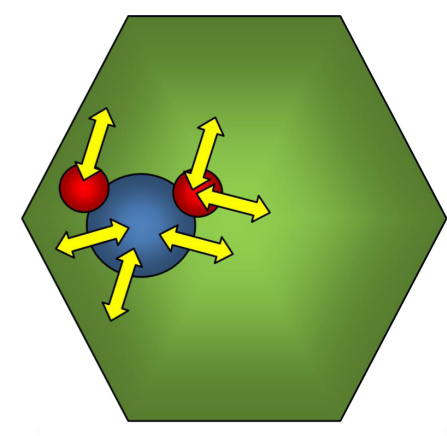

Water Molecule Solution in 3D $\mathrm{CO}_{2}$ Cluster

Figure 5. Three types of water solution in gaseous $\mathrm{CO}_{2}$ : blue balls, oxygen atoms; red balls, hydrogen atoms; green ball, carbon atom; green hexagonal, three-dimensional $\mathrm{CO}_{2}$ cluster; yellow arrows, interatomic bonds.

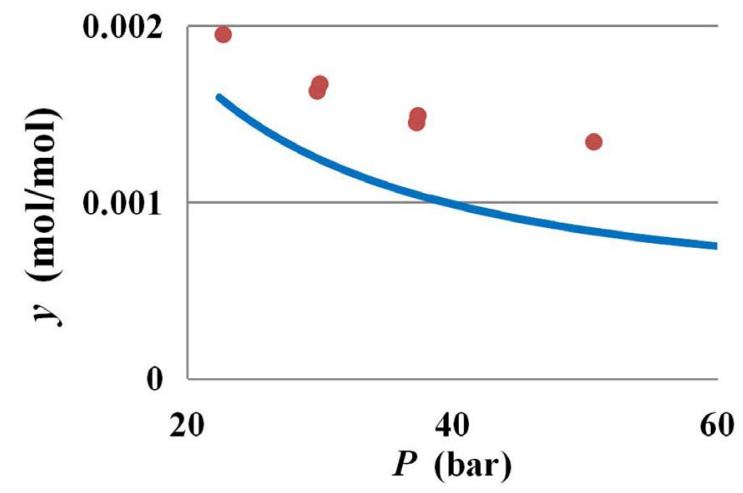

Figure 6. Water solubility $y$ in gaseous $\mathrm{CO}_{2}$ according to experimental data from [36] (markers) and theoretical free space solubility $y_{f s}$ (solid blue line) at subcritical temperature $25^{\circ} \mathrm{C}$.

$R T / P$. In real gaseous solutions, $V_{f s}(T, P)$ deviates from the ideal gas value. In a real gas, the free space for the distribution of solute molecules is created by freely moving particles of a different nature: monomers and clusters of the solvent, solute molecules themselves, and the solute-solvent clusters. In real gaseous solutions, for $V_{f}$, instead of using the solvent's molar volume $V_{s}$, it is more correct to use the free moving particles' molar volume $V_{p}=1 / D_{p}$, where $D_{p}=P / R T$ is the free moving particles' density, responsible for pressure in a real gas [40]. Figure 7 shows the values of the free moving particles' molar density $D_{p}$ in gaseous $\mathrm{CO}_{2}$ at $25^{\circ} \mathrm{C}$, in comparison with the total molar density $D$ and the monomer fraction density $D_{m}$. The difference between $D$ and $D_{p}$ is due to the clusters' contribution to the total density.

\subsection{Water Solubility in Gaseous $\mathrm{CO}_{2}$ at Pressures below $\boldsymbol{P}_{c}$ and above $\boldsymbol{P}_{c} / 2$}

Figure 6 shows the comparison of the free space solubility $y_{f s}$ of water in $\mathrm{CO}_{2}$ with experimental solubility data at subcritical pressures of the solvent. This figure is typical for many solute-solvent systems at pressures lower than the critical 


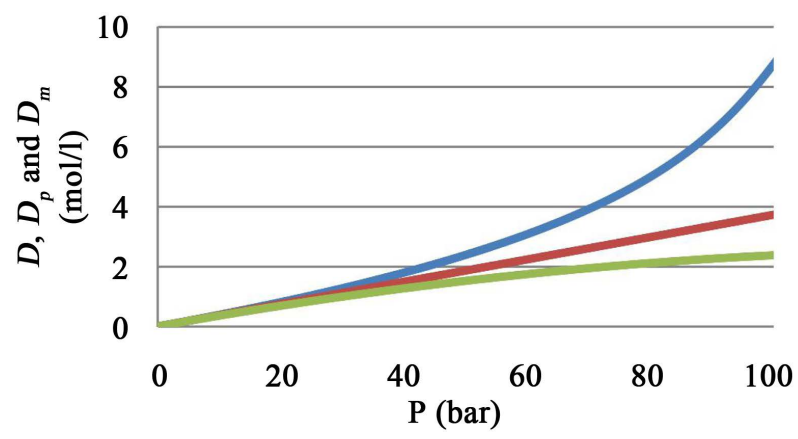

Figure 7. Total molar density $D$ values taken from [13] (solid blue line), free moving particles' density $D_{p}$ (dashed red line), and monomer fraction density $D_{m}$ (dotted green line) in $\mathrm{CO}_{2}$ at $25^{\circ} \mathrm{C}$.

pressure. It shows that the experimental solubility data at a constant temperature differ from the free space solubility on the close to constant value $\Delta y(P)$. In the pressure range of almost constant $\Delta y(P)$ and at a fixed temperature $T$, we can estimate the average value $\Delta y_{a r}(T)$. This value is the number of solute moles bound with one mole of solvent molecules by molecular cross-interaction forces (central type in Figure 5). This increment over the free space solubility may be essential, especially close to critical pressures.

The $\Delta y_{a v}(T)$ to $D_{w s a t}(T)$ ratio is the equilibrium constant for the solute-solvent cross-interaction $C_{w-c d}$, reflected in Table 3 for the $\mathrm{H}_{2} \mathrm{O}-\mathrm{CO}_{2}$ system. For the total water solubility $y(T, P)$ in $\mathrm{CO}_{2}$ at pressures $P<P_{\circ}$ we have the following:

$$
y(T, P)=D_{\text {wsat }}(T)\left(R T / P+C_{w-c d}\right) .
$$

The corresponding data for two other systems that were analyzed are presented in Table 4.

The expression (7) reflects both the solution's colligative properties, reflected in $R T / P$, and individual properties of the solution system, reflected in the solute-solvent cross-interaction equilibrium constant $C_{w-c d}$.

Table 3 and Table 4 show that the equilibrium constants for the cross-interaction of water molecules in gaseous solutions with $\mathrm{CO}_{2}$ and $\mathrm{N}_{2} \mathrm{O}$ molecules are of the same order of magnitude, but with $\mathrm{C}_{2} \mathrm{H}_{6}$ molecules they are much smaller. The temperature dependence of equilibrium constants permits us to estimate the cross-interaction parameters: the bond energy $E_{\text {cross }}$ and the corresponding entropic factor, and the effective volume of molecular cross-interaction

$$
V_{\text {cross }}=C_{\text {cross }} / \exp \left(E_{\text {cross }} / T\right) \text {, }
$$

these are reflected in Table 5. To find these parameters, we plot $\ln \left(C_{\text {cross }}\right)$ versus $1000 / T$ (see Figure 8). The linear trend for the experimental data provides the required parameters.

We see that despite a zero dipole moment value for the $\mathrm{CO}_{2}$ molecule, its cross-interaction bond energy $E_{\text {cross }}$ with polar water molecules is rather high due to the existence of the quadrupole moment [38] in the $\mathrm{CO}_{2}$ molecule. Hence, the atoms in this molecule are charged. 
Table 3. The saturated water vapor density $D_{w s a t}$ according to [13], the averaged solubility increment $\Delta y_{a v}$ over the free space solubility and the equilibrium constant $C_{W-c d}$ for the water-carbon dioxide dimer fraction.

\begin{tabular}{cccc}
\hline$T$ & $D_{\text {wsat }}$ & $\Delta y_{\text {aV }}$ & $C_{\text {w-cd }}$ \\
\hline $\mathrm{K}$ & $(\mathrm{mol} / \mathrm{l})$ & $(\mathrm{mol} / \mathrm{mol})$ & $(1 / \mathrm{mol})$ \\
\hline 298,15 & 0.0013 & 0.00057 & 0.443 \\
323,15 & 0.0046 & 0.00114 & 0.246 \\
348,15 & 0.0135 & 0.00249 & 0.185 \\
373,15 & 0.0332 & 0.00428 & 0.129 \\
\hline
\end{tabular}

Table 4. The solubility increment $\Delta y_{a v}$ over the free space solubility, the equilibrium constants $C_{W-n o}$ and $C_{w-e t h}$ for the water-nitrous oxide and water-ethane dimers.

\begin{tabular}{ccccc}
\hline & \multicolumn{3}{c}{ Systems } \\
\hline Temperature & \multicolumn{2}{c}{ Water-nitrous oxide } & \multicolumn{2}{c}{ Water-ethane } \\
\hline$T$ & $\Delta y_{a V}$ & $C_{W-n o}$ & $\Delta y_{a V}$ & $C_{W-\text { eth }}$ \\
\hline $\mathrm{K}$ & $(\mathrm{mol} / \mathrm{mol})$ & $(\mathrm{l} / \mathrm{mol})$ & $(\mathrm{mol} / \mathrm{mol})$ & $(1 / \mathrm{mol})$ \\
\hline 298.15 & 0.00047 & 0.364 & 0.000049 & 0.038 \\
323.15 & 0.00111 & 0.241 & 0.000094 & 0.02 \\
348.15 & 0.00238 & 0.177 & 0.000197 & 0.015 \\
373.15 & 0.00455 & 0.137 & 0.001025 & 0.031 \\
\hline
\end{tabular}

Table 5. The estimated cross-interaction parameters: bond energy $E_{\text {cross }}$ and effective cross interaction molar volume $V_{\text {cross. }}$.

\begin{tabular}{ccc}
\hline System & $E_{\text {cross }}(\mathrm{K})$ & $V_{\text {cross }}(1 / \mathrm{mol})$ \\
\hline $\mathrm{H}_{2} \mathrm{O}-\mathrm{CO}_{2}$ & 1783.5 & 0.0011 \\
$\mathrm{H}_{2} \mathrm{O}-\mathrm{N}_{2} \mathrm{O}$ & 1441.7 & 0.0028 \\
$\mathrm{H}_{2} \mathrm{O}-\mathrm{C}_{2} \mathrm{H}_{6}$ & 510 & 0.0053 \\
\hline
\end{tabular}

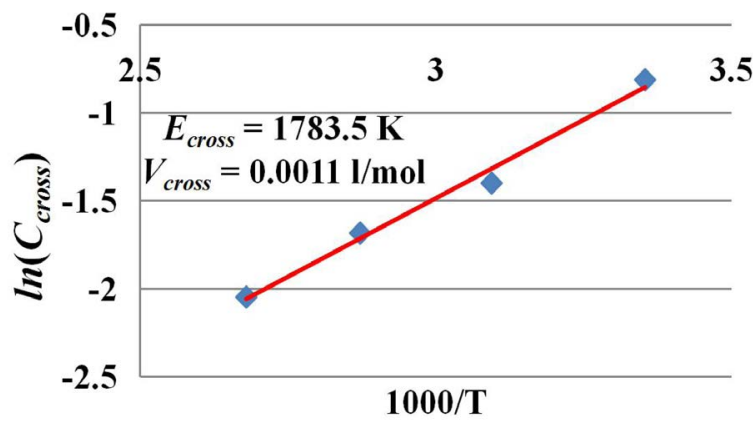

Figure 8. Estimation of cross-interaction parameters for the $\mathrm{H}_{2} \mathrm{O}-\mathrm{CO}_{2}$ system: cross-interaction bond energy $E_{W-c d}$ and effective cross-interaction molar volume $V_{w-c d}$.

A high cross-interaction bond energy $E_{\text {cross }}$ in the $\mathrm{H}_{2} \mathrm{O}-\mathrm{N}_{2} \mathrm{O}$ system results from a combination of dipole and quadrupole potentials of the $\mathrm{N}_{2} \mathrm{O}$ molecule. 
As Table 1 shows, the dipole moment in the $\mathrm{N}_{2} \mathrm{O}$ molecule is not very large.

A symmetric ethane molecule possesses no dipole or quadrupole moments. But its cross-interaction bond energy with the water molecule is high enough, although not as high as in $\mathrm{H}_{2} \mathrm{O}-\mathrm{CO}_{2}$ and $\mathrm{H}_{2} \mathrm{O}-\mathrm{N}_{2} \mathrm{O}$ systems. The cross-bond energy in the $\mathrm{H}_{2} \mathrm{O}-\mathrm{C}_{2} \mathrm{H}_{6}$ system results from the multipole moment [38] of the $\mathrm{C}_{2} \mathrm{H}_{6}$ molecule, caused by no zero atoms' charges in this molecule, as discussed in [39].

\subsection{Water Solubility in $\mathrm{CO}_{2}$ at Supercritical Pressures}

In the third type of solution, in three-dimensional (3D) clusters, the number of water molecular bonds with surrounding $\mathrm{CO}_{2}$ molecules increases (see Figure 5). This results in an increase in water solubility with pressure in high-density gaseous $\mathrm{CO}_{2}$ (see Figure 9).

Figure 9 demonstrates that at $75^{\circ} \mathrm{C}$ and $P<40$ bar, the solubility increment $\Delta y(P)$ is almost constant according to the second type of solubility; however, at $P>40$ bar, the increment essentially increases with pressure. Similar behavior of $\Delta y(P)$ is seen at $50^{\circ} \mathrm{C}$ (see Figure 4).

We now consider the solubility increment $\Delta_{3 \mathrm{D}} y$ caused only by the $3 \mathrm{D}$ clusters, by subtracting from the total solubility $y$ the estimated values for the second type of solubility mechanism. According to [16], at supercritical temperatures the number of $3 \mathrm{D}$ clusters rapidly increases as the total density $D$ approaches the critical density.

It is possible to estimate approximately the number of particles in $3 \mathrm{D}$ clusters, providing maximum contribution to the solubility increment at supercritical pressures. For this we plot the $\ln \left(\Delta_{3 \mathrm{D}} y\right)$ versus $\ln \left(D_{m}\right)$ (see Figure 10) and estimate the tangent of the slope for the linear trend of the graph, $K_{3 D}$ :

The tangent of the slope for the graph is close to 7. Hence the 7-particle clusters provide the maximum contribution to the water solubility in $\mathrm{CO}_{2}$ at $50^{\circ} \mathrm{C}$ and pressures up to $100 \mathrm{bar}$. The proportionality factor $k_{3 D}$ between $\Delta_{3 \mathrm{D}} y$ and $D_{m}^{7}$ is $4.91 \times 10^{-6}(1 / \mathrm{mol})^{7}$ (see Figure 11$)$.

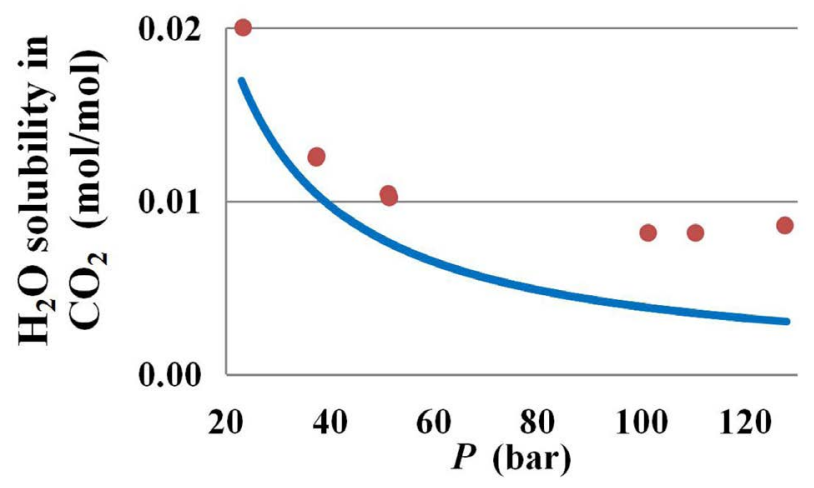

Figure 9. Water solubility increase at supercritical pressures in carbon dioxide at $75^{\circ} \mathrm{C}$ : experimental data from [36] (markers) and theoretical solubility in a free space of a gaseous solution (solid blue line). 


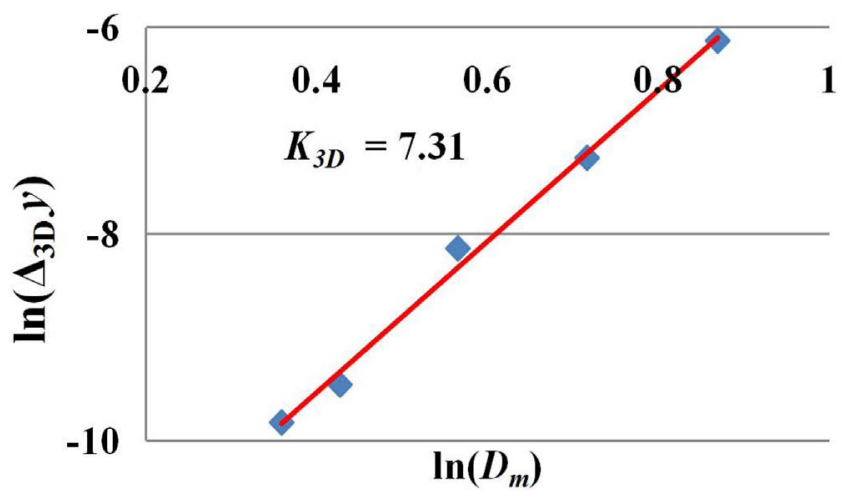

Figure 10. Estimation of the particle numbers in $3 \mathrm{D}$ clusters at $50^{\circ} \mathrm{C}$ and supercritical pressures, leading to an increase in water solubility in $\mathrm{CO}_{2}$, by plotting the logarithm of the $3 \mathrm{D}$ solubility increment versus the logarithm of the monomer fraction density $D_{m}$.

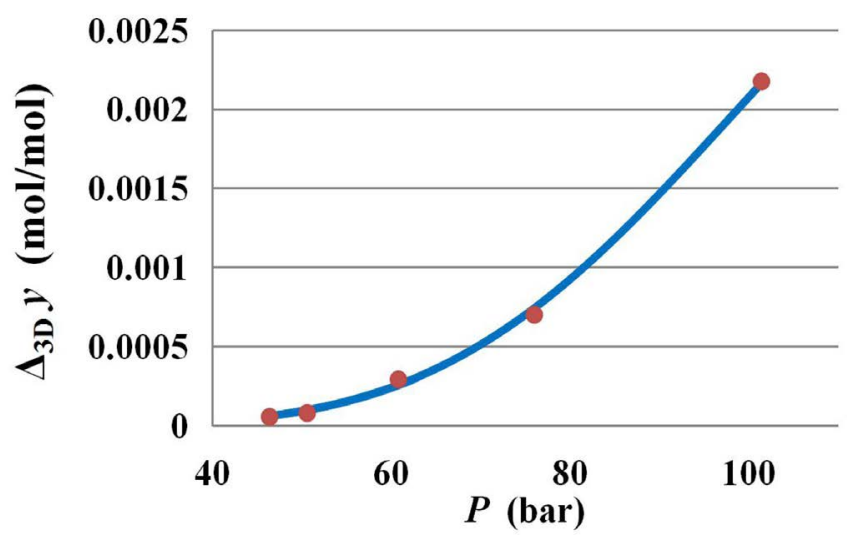

Figure 11. Increase in water solubility increment in supercritical $\mathrm{CO}_{2}$ at $50^{\circ} \mathrm{C}$, caused by $3 \mathrm{D}$ clusters: $\Delta_{3 \mathrm{D}} y$ (markers) and its model, $\Delta_{3 \mathrm{D}} y_{\text {mod }}=k_{3 D} \times D_{m}^{7}($ solid blue line $) ; k_{3 D}=4.91 \times 10^{-6}$ $(\mathrm{l} / \mathrm{mol})^{7}$.

\subsection{Solubility of Ethanol in Sub- and Supercritical $\mathrm{CO}_{2}$}

The theory of three types of solubility developed for the water- $\mathrm{CO}_{2}$ system may be applied to the ethanol- $\mathrm{CO}_{2}$ system. The ethanol solubility $y(T, P)$ in gaseous $\mathrm{CO}_{2}$ has been calculated based on theoretical estimations from [19] for the $\mathrm{CO}_{2}$ molar share in a gaseous ethanol- $\mathrm{CO}_{2}$ system at temperatures of $25^{\circ} \mathrm{C}, 40^{\circ} \mathrm{C}$, $45^{\circ} \mathrm{C}$, and $50^{\circ} \mathrm{C}$. In [19], the authors used four theoretical models and achieved the best performance with the Peng-Robinson/Mathias-Klotz-Prausnitz approach [41] to estimate the molar share of $\mathrm{CO}_{2}$ in the gaseous $\mathrm{CO}_{2}$-ethanol system. Figure 12 demonstrates a good qualitative correspondence of the ethanol solubility pressure dependence with the theory of three types of solubility for polar substances in sub- and supercritical $\mathrm{CO}_{2}$.

Figure 12 shows an almost constant value for the solubility increment $\Delta y$ at $\mathrm{P}<55$ bar and the solubility minimum near $\mathrm{P}=60$ bar. Comparison of Figure 12 with Figure 4 shows that the solubility of ethanol in gaseous $\mathrm{CO}_{2}$ at $\mathrm{P}>30$ 


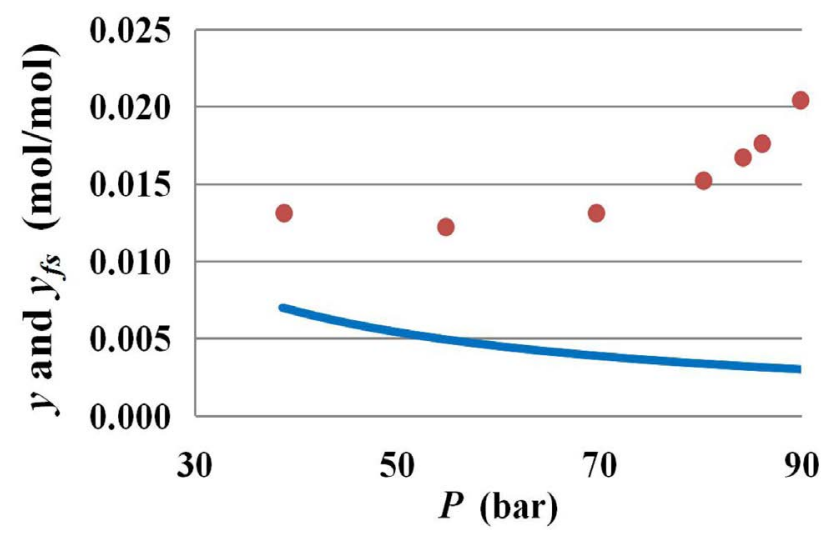

Figure 12. Pressure dependence of the ethanol solubility $y$, based on data from [19] (markers), in comparison with the free space solubility $y_{f s}$ (solid blue line) in supercritical $\mathrm{CO}_{2}$ at $50^{\circ} \mathrm{C}$.

bar is greater than the solubility of water at the same temperature and pressure. This is due to the ethanol vapor density being double that of the water vapor density under the same conditions. Furthermore, the solubility increment $\Delta y$ for the ethanol- $\mathrm{CO}_{2}$ system is larger than for the water- $\mathrm{CO}_{2}$ system and larger than the free space solubility $y_{f s}$. This indicates that the equilibrium constant for the ethanol- $\mathrm{CO}_{2}$ dimers is larger than for the $\mathrm{H}_{2} \mathrm{O}-\mathrm{CO}_{2}$ dimers. For a detailed analysis of the cross-interactions in the ethanol- $\mathrm{CO}_{2}$ dimers, more precise experimental solubility data are required.

\subsection{Solubility of Ethanol in Liquid-Like Sub- and Supercritical $\mathrm{CO}_{2}$}

In Section 1.3, the unwanted transformation in chromatography columns of a liquid-like SF to a condensed gas under high pressure was described. This transformation may occur when the pressure falls to the bubble pressure (discussed in [19]). At the point of this transformation in the chromatography column with a $\mathrm{CO}_{2}$-modifier system, the modifier's molar share may be different for the liquid-like and gaseous sections. Under equilibrium conditions, at the bubble pressure, from the two-component liquid-like SF system in the gaseous bubble enters mainly $\mathrm{CO}_{2}$, and the modifier's molar share $y_{g a s}$ in the bubble becomes much lower than $y_{l i q}$ in the liquid-like section. This is illustrated in Figure 13, built on the basis of data from [19], where $y_{g a s}$ is multiplied by a factor of 100 to compare it with the $y_{i q}$ values at the same pressure.

Figure 13 shows that the $20 \%-30 \%$ modifier content in the liquid $\mathrm{CO}_{2}$ ethanol section of the chromatography column may be transformed to the gas phase, with only $1 \%$ of modifier in it. A similar process takes place at a subcritical temperature (see Figure 14).

Here again we see that the $20 \%-30 \% \mathrm{CO}_{2}$-ethanol liquid section in the chromatography column may be transformed in the bubble point to a $0.4 \% \mathrm{CO}_{2^{-}}$ ethanol gaseous system. The content of the analyte may differ significantly from its content in the liquid section, which may distort the chromatography results. 


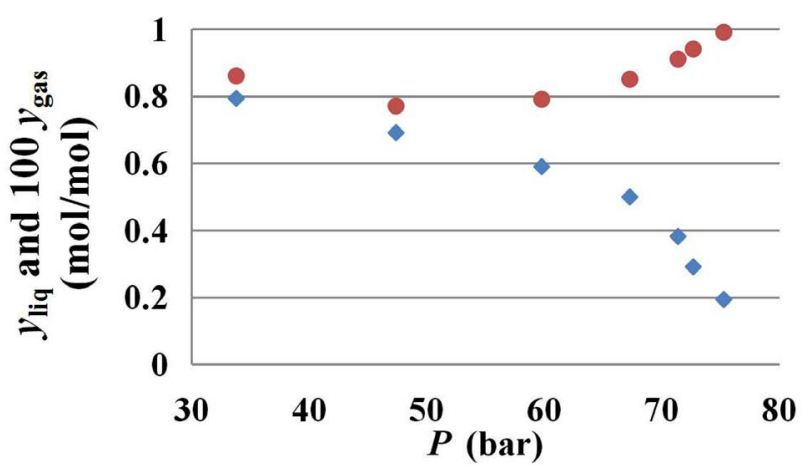

Figure 13. Pressure dependence of ethanol molar share in the liquid-like (blue rhombs) and gaseous (red circles) SF $\mathrm{CO}_{2}-$ ethanol systems at equilibrium in the bubble point at supercritical temperature $40^{\circ} \mathrm{C}$. Data are based on the results of [19].

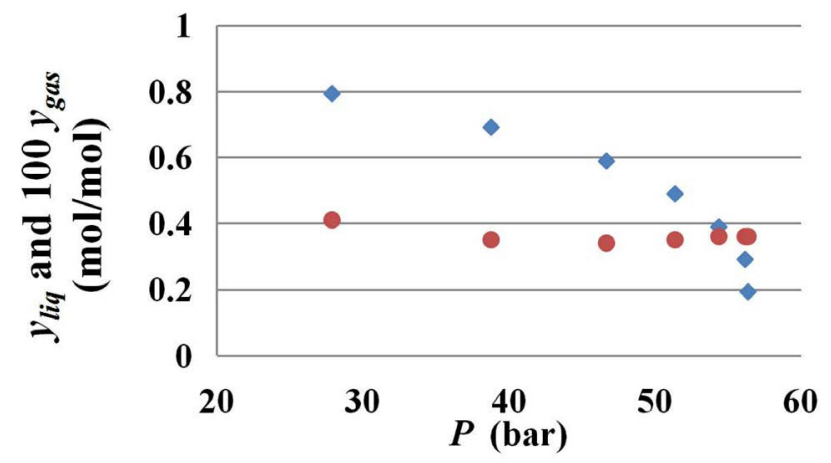

Figure 14. Pressure dependence of ethanol molar share in the liquid (blue rhombs) and gaseous (red circles) $\mathrm{CO}_{2}$-ethanol systems at equilibrium in the bubble point at subcritical temperature $25^{\circ} \mathrm{C}$. Data are based on the results of [19].

\section{Possible Influence of Melting and Triple Point Temperature Harmonic Zigzag Oscillations of a Homologous Series of $\boldsymbol{n}$-Alkanoic Substances with Different Numbers of Carbon Atoms}

$\mathrm{N}$-alkanoic substances are widely used in SF technologies both as the subjects of complex mixtures for analyzing purposes, as principal mobile phases, and as modifiers. Formic acid is an important substance in the performance of negative MS in cases when positive MS fails to produce decisive evidence for the mass ion [42] [43]. This acid has a very low response in a flame ionization detector (FID), permitting us to use it as a modifier in SFC coupled with FID [44]. In HPLC, formic acid is commonly used for buffer preparation and $\mathrm{pH}$ regulation. This also applies to acetic acid. Besides $n$-alkanoic acids, $n$-alcohols and amines are often used in SFC as modifiers [45]. The influence of modifiers' individual properties on solubility has a significant impact on extraction and bond separation; this is described in detail in [46].

Our previous work described the phenomenon of harmonic zigzag oscillations of melting and triple point temperatures for members of a homologous series 
with different numbers of carbon atoms $\left(N_{C}\right)$ in $n$-alkanes, $n$-alkanoic acids, $n$-alcohols, and in some families of other homologous series of organic substances [39]. The melting and triple point temperatures of substances with even $N_{C}$ in their chains are higher than the average values of similar substances with the nearest odd numbers. This phenomenon exists in many homologous series and may influence the solubility power of the SF mobile phase with organic modifiers. The solubility power cannot be related to colligative properties because it depends on individual molecular interaction properties of the substances used.

Because of harmonic zigzag progression of temperatures of melting and triple points, it could be of interest to determine the influence on pressure-temperature relations (bar/Kelvin, K) diagrams of three successive members of homologous series after the addition of a range of substances to the supercritical mobile phase, as modifiers to $\mathrm{SCCO}_{2}$ (substances include $n$-pentane, $n$-hexane, and, $n$-heptane; $n$-pentanol, $n$-hexanol, and $n$-heptanol; and $n$-pentanoic, $n$-hexanoic, and $n$-heptanoic acids).

We previously described the tangent of the slope change of the melting temperature progression of members of a homologous series before the $N c 5$ and after the $N c 5$ [39]. The use of these members in a homologous series may also influence the properties of $\mathrm{SCCO}_{2}$ with organic modifiers. It may be of further interest to explore the effect of the addition of methanol and ethanol as modifiers to $\mathrm{SCCO}_{2}$ on pressure-temperature differences. Although qualitative calculations for some systems, $n$-butane [42], (water $+n$-butane) and (water $+n$-hexane) [47], could show some deviations from $\mathrm{CO}_{2}$ data, they are useful to see the influence (qualitative only) of different species in a homologous series with different $N_{C}$.

Overall, it is important to understand the physical-chemical and thermodynamic aspects of deviation of the first members of a homologous series from regular dependence.

\section{Discussion}

The mechanism of water and ethanol solubility in dense gaseous $\mathrm{CO}_{2}$ at sub- and supercritical temperatures, as considered here, is common to many different gaseous solvent-solute systems, if the solute vapor pressure is high enough, as in the case of ethanol. At low solute vapor pressure, the first two types of solution mechanism discussed are not essential; it is the third type of solution that dominates, as in the case of anthracene or phenanthrene, which are widely used in investigations into solubility in $\mathrm{SCCO}_{2}[42]$.

Extension of the theory of three types of solution mechanism suggested here to a wide series of solute-solvent high-pressure gaseous systems requires more accurate data on the solubility at sub- and supercritical temperatures and pressures.

The tangent of the slope change of melting temperature progression may have an influence on the choice of modifier (methanol versus ethanol, or methanol/ 
ethanol versus higher alcohols), in relation to the pressure-temperature diagram (see Figure 3) and results of separation, resolution of bonds in SFC, or quantitative results of extraction by SFE. The tangent of the slope change for $N_{C}$ below and above $N_{C} 5$ was observed in all homologous series described in [39]. The choice of a substance from a homologous series with $N_{C}$ below or above $N_{C} 5$ may dramatically change the results of the procedure.

\section{References}

[1] Poole, C.F. (2000) Progress in Packed Column Supercritical Fluid Chromatography: Materials and Methods. Journal of Biochemical and Biophysical Methods, 43, 3-23. https://doi.org/10.1016/S0165-022X(00)00057-9

[2] Sjöberg, P.J.R. and Markides, K.E. (1999) Capillary Column Supercritical Fluid Chromatography-Atmospheric Pressure Ionization Mass Spectrometry: Interface Performance of Atmospheric Pressure Chemical Ionization and Electrospray Ionization. Journal of Chromatography A, 855, 317-327. https://doi.org/10.1016/S0021-9673(99)00675-5

[3] Baker, T.R. and Pinkston, J.D. (1998) Development and Application of Packed Column Supercritical Fluid Chromatography/Pneumatically Assisted Electrospray Mass Spectrometry. Journal of the American Society for Mass Spectrometry, 9, 498-509. https://doi.org/10.1016/S1044-0305(98)00006-3

[4] Schweighardt, F.K. and Mathias, P.M. (1993) Impact of Phase Equilibria on the Behavior of CylinderStored $\mathrm{CO}_{2}$-Modifier Mixtures Used as Supercritical Fluid. Journal of Chromatographic Science, 31, 207-211. https://doi.org/10.1093/chromsci/31.6.207

[5] Brondz, I. and Høiland, K. (2008) Chemotaxonomic Differentiation between Cortinarius infractus and Cortinarius subtortus by Supercritical Fluid Chromatography Connected to a Multi-Detection System. Trends in Chromatography, 4, 79-87.

[6] Saito, M. (2013) History of Supercritical Fluid Chromatography: Instrumental Development. Journal of Bioscience and Bioengineering, 115, 590-599. https://doi.org/10.1016/j.jbiosc.2012.12.008

[7] Brondz, I. (2012) Editorial: Yesterday, Today and Tomorrow of Supercritical Fluid Extraction and Chromatography. American Journal of Analytical Chemistry, 3, 867 -869. https://doi.org/10.4236/ajac.2012.312A114

[8] Brondz, I. and Brondz, A. (2014) Review: Isomer Separation, Chiral Resolution, and Structure Elucidation Analyses Are the Future of Analytical Supercritical Fluid Chromatography-Mass Spectrometry. International Journal of Analytical Mass Spectrometry and Chromatography, 2, 15-24.

https://doi.org/10.4236/ijamsc.2014.21002

[9] Brondz, I. and Brondz, A. (2012) The Technology for Preparation of Generic (Monoenantiomeric) Antimalarial Drug Primaquine by Using Supercritical Fluid Chromatography. Separation of Primaquine from Quinocide: Simultaneous Resolution of the Enantiomers of Primaquine and Their Separation from Quinocide in One Run. American Journal of Analytical Chemistry, 3, 884-890. https://doi.org/10.4236/ajac.2012.312A117

[10] Brondz, I., Høiland, K. and Lefler, J. (2007) Supercritical Fluid Chromatography of Secondary Metabolites and Multi-Analysis by Mass Spectrometry-Ultraviolet and Corona Charged Aerosol Detection. 12 Norwegian MS-Winter Meeting, Hafjell, 21-24 January 2007, 63.

[11] Pitchaiah, K.C., Brahmananda Rao, C.V.S., Sivaraman, N., Joseph, M. Madras, G. and 
Brondz, I. (2017) Solubility of Dialkylalkyl Phosphonates in Supercritical Carbon Dioxide: Experimental and Modeling Approach. Fluid Phase Equilibria, 435, 88 -97. https://doi.org/10.1016/j.fluid.2016.12.011

[12] Weast, R.C. (1984) CRC Handbook of Chemistry and Physics. Chemical Rubber Company, Boca Raton.

[13] NIST Chemistry WebBook (2016) Thermophysical Properties of Fluid Systems. http://webbook.nist.gov/chemistry/fluid

[14] NIST Chemistry WebBook (2016) NIST Standard Reference Database Number 69. http://webbook.nist.gov/chemistry

[15] Sedunov, B. (2013) The Equilibrium Thermal Physics of Supercritical Fluids. International Journal of Analytical Mass Spectrometry and Chromatography, 1, 103-108. https://doi.org/10.4236/ijamsc.2013.12013

[16] Sedunov, B. Brondz, I. (2016) Analytical Approach to Clusters in near Critical $\mathrm{CO}_{2}$. International Journal of Analytical Mass Spectrometry and Chromatography, 4, 3950. https://doi.org/10.4236/ijamsc.2016.43005

[17] Levy, M. and Ritchey, W.M. (1985) The Effects of Modifiers in Supercritical Fluid Chromatography. Journal of High Resolution Chromatography, 8, 503-509. https://doi.org/10.1002/jhrc.1240080903

[18] Brondz, I. and Brondz, A. (2012) Supercritical Fluid Chromatography-Mass Spectrometry (SFC-MS) of Heterocyclic Compounds with Trivalent and Pentavalent Nitrogen in Cough Relief Medical Forms Tuxi and Cosylan. American Journal of Analytical Chemistry, 3, 870-876. https://doi.org/10.4236/ajac.2012.312A115

[19] Mehl, A., Nascimento, F.P., Falcão, P.W., Pessoa, F.L.P. and Cardozo-Filho, L. (2011) Vapor-Liquid Equilibrium of Carbon Dioxide + Ethanol: Experimental Measurements with Acoustic Method and Thermodynamic Modeling. Journal of Thermodynamics, 2011, $11 \mathrm{p}$.

[20] Gernert, J. Jäger, A. and Span, R. (2014) Calculation of Phase Equilibria for Multy-Component Mixtures Using Highly Accurate Helholtz Energy Equations of State. Fluid Phase Equilibria, 375, 209-218. https://doi.org/10.1016/j.fluid.2014.05.012

[21] Chueh, P.L. and Prausnitz, J.M. (1967) Vapor-Liquid Equilibria at High Pressures: Calculation of Critical Temperatures, Volumes, and Pressures of Nonpolar Mixtures. American Institute of Chemical Engineers, 13, 107-1113. https://doi.org/10.1002/aic.690130612

[22] Chueh, P. L. Prausnitz, J. M. (1967), Vapor-Liquid Equilibria at High-Pressures: Calculation of Partial Molar Volumes in Nonpolar Liquid Mixtures. American Institute of Chemical Engineers, 13, 1099-1107.

http://onlinelibrary.wiley.com/doi/10.1002/aic.690130612/abstract

[23] Gupta, R.B., Shim, J.-J. (2006) Solubility in Supercritical Carbon Dioxide. CRC Press, Taylor \& Francis Group, Abingdon, 960.

[24] Hawthorne, S.B. (1990) Analytical-Scale Supercritical Fluid Extraction. Analytical Chemistry, 62, 633A-642A. https://doi.org/10.1021/ac00210a722

[25] Kuei, J.C., Markides, K.E. and Lee, M.L. (1987) Supercritical Ammonia as Mobile Phase in Capillary Chromatography. Journal of Separation Science, 10, 257-262. https://doi.org/10.1002/jhrc.1240100509

[26] Brunner, E. Hultenschmidt, W. and Schlichtharle, G. (1987) Fluid Mixtures at High Pressures IV. Isothermal Phase Equilibria in Binary Mixtures Consisting of Methanol + Hydrogen or Nitrogen or Methane or Carbon Monoxide or Carbon Dioxide. The Journal of Chemical Thermodynamics, 19, 273-291. https://doi.org/10.1016/0021-9614(87)90135-2 
[27] Lin, Y. Wai, C.M. Jean, F.M. and Brauer, R.D. (1994) Supercritical Fluid Extraction of Thorium and Uranium Ions from Solid and Liquid Materials with Fluorinated $\beta$-Diketones and Tri-Butyl Phosphate. Environmental Science \& Technology, 28, 1190-1193. https://doi.org/10.1021/es00055a034

[28] Lin, Y., Brauer, R.D., Laintz, K.E. and Wai, C.M. (1993) Supercritical Fluid Extraction of Lanthanides and Actinides from Solid Materials with a Fluorinated B-Diketone. Analytical Chemistry, 65, 2549-2551. https://doi.org/10.1021/ac00066a027

[29] Lin, Y. and Wai, C.M. (1994) Supercritical Fluid Extraction of Lanthanides with Fluorinated $\beta$-Diketones and Tri-Butyl Phosphate. Analytical Chemistry, 66, 1971 1975. https://doi.org/10.1021/ac00085a008

[30] Kumar, R., Sivaraman, N., Senthil Vadivu, E., Srinivasan, T.G., Vasudeva Rao, P.R. (2003) Complete Removal of Uranyl Nitrate from Tissue Matrix Using Supercritical Fluid Extraction. Radiochimica Acta, 91, 197-201. https://doi.org/10.1524/ract.91.4.197.19966

[31] Shimad, T., Ogumo, S., Sawada, K., Enokida, Y. and Yamamato, Y. (2006) Selective Extraction of Uranium from a Mixture of Metal or Metal Oxides by a Tri- $n$-Butylphosphate Complex with $\mathrm{HNO}_{3}$ and $\mathrm{H}_{2} \mathrm{O}$ in Supercritical $\mathrm{CO}_{2}$. Analytical Sciences, 22, 1387-1391. https://doi.org/10.2116/analsci.22.1387

[32] Lin, Y., Liu, C., Wu, H., Yak, H.K. and Wai, C.M. (2003) Supercritical Fluid Extraction of Toxic Heavy Metals and Uranium from Acidic Solutions with Sulfur Containing Organophosphorous Reagents. Industrial \& Engineering Chemistry Research, 42, 1400-1405. https://doi.org/10.1021/ie020804i

[33] Kumar, R. Sivaraman, N. Sujatha, K. Srinivasan, T.G. and Vasudeva Rao, P.R. (2007) Removal of Plutonium and Americium from Waste Matrices by Supercritical Carbon Dioxide Extraction. Radiochimica Acta, 95, 577-584. https://doi.org/10.1524/ract.2007.95.10.577

[34] Erkey, C. (2000) Supercritical Carbon Dioxide Extraction of Metals from Aqueous Solutions: A Review. Journal of Supercritical Fluids, 17, 259-287. https://doi.org/10.1016/S0896-8446(99)00047-9

[35] Pauling, L. (1970) General Chemistry. W.H. Freeman and Co., San-Francisco.

[36] Coan, C.R. and King, A.D. (1971) Solubility of Water in Compressed Carbon Dioxide, Nitrous Oxide, and Ethane. Evidence for Hydration of Carbon Dioxide and Nitrous Oxide in the Gas Phase. Journal of the American Chemical Society, 93, 1857. https://doi.org/10.1021/ja00737a004

[37] Wiebe, R. (1941) The Binary System Carbon Dioxide-Water under Pressure. Chemical Reviews, 29, 475-481. https://doi.org/10.1021/cr60094a004

[38] Stogryn, D.E. and Stogryn, A.P. (1966) Molecular Multipole Moments. Molecular Physics, 11, 371-393. https://doi.org/10.1080/00268976600101201

[39] Sedunov, B. and Brondz, I. (2017) The Zigzag Progression of Melting and Triple Point Properties of $n$-Alkanes, $n$-Alcohols, $n$-Alkanoic Amines and $n$-Alkanoic Acids. Voice of the Publisher, 3, 1-14. https://doi.org/10.4236/vp.2017.31001

[40] Sedunov, B. (2008) Monomer Fraction in Real Gases. International Journal of Thermodynamics, 11, 1-9.

[41] Mathias, P.M., Klotz, H.C. and Prausnitz, J.M. (1991) Equation-of-State Mixing Rules for Multicomponent Mixtures: the Problem of Invariance, Fluid Phase Equilibria, 67, 31-44. https://doi.org/10.1016/0378-3812(91)90045-9

[42] McHugh, M.A. and Krukonis, V.J. (1994) Supercritical Fluid Extraction: Principles and Practice. Butterworth-Heinemann, Boston. 
http://www.phasex4scf.com/supercritical-fluids/supercritical-fluids-publications-pa pers

[43] Señoráns, F.J. and Ibañez, E. (2002) Review: Analysis of Fatty Acids in Foods by Supercritical Fluid Chromatography. Analytica Chimica Acta, 465, 131-144. https://doi.org/10.1016/S0003-2670(02)00208-8

[44] JASCO SFC-FID (2015) Editorial: Fuel Analysis, https://jascoinc.com/wp-content/uploads/2015/05/Evaluation-of-the-SFC-FID-for-t he-Olefin-Analysis-ASTM-D6550-Method.pdf

[45] Gumerov, F.M., Sagdeev, A.A. and Amirkhanov, D.G. (2016) The Solubility of Substances in Supercritical Fluid Media. LAP LAMBERT Academic Publishing GmbH \& Co. KG., Saarbrucken, 336 p.

[46] Brondz, I., Ekeberg, D., Bell, D.S., Hustad, J.A., Svendsen, R., Vlachos, V., Oakley, P., Langley, G.J., Mohini, T. and Amaury, C-G. Mikhalitsyn, F. (2007) Nature of the Main Contaminant in the Drug Primaquine Diphosphate: SFC and SFC-MS Methods of Analysis. Journal of Pharmaceutical and Biomedical Analysis, 43, 937-944. https://doi.org/10.1016/j.jpba.2006.09.017

[47] Yiling, T. Michelberger, Th. and Franck, E.U. (1991) High-Pressure Phase Equilibria and Critical Curves of (Water $+n$-Butane) and (Water $+n$-Hexane) at Temperatures to $700 \mathrm{~K}$ and Pressures to $300 \mathrm{MPa}$. The Journal of Chemical Thermodynamics, 23, 105-112. https://doi.org/10.1016/S0021-9614(05)80063-1

\section{Scientific Research Publishing}

\section{Submit or recommend next manuscript to SCIRP and we will provide best} service for you:

Accepting pre-submission inquiries through Email, Facebook, LinkedIn, Twitter, etc. A wide selection of journals (inclusive of 9 subjects, more than 200 journals) Providing 24-hour high-quality service User-friendly online submission system Fair and swift peer-review system Efficient typesetting and proofreading procedure Display of the result of downloads and visits, as well as the number of cited articles Maximum dissemination of your research work

Submit your manuscript at: http://papersubmission.scirp.org/ Or contact ijamsc@scirp.org 